\title{
Information extraction framework for Kurunthogai
}

\author{
C N SUBALALITHA® \\ Department of Computer Science and Engineering, SRM Institute of Science and Technology, \\ Kattankulathur 603 203, India \\ e-mail: subalalitha@gmail.com
}

MS received 12 August 2017; revised 9 March 2019; accepted 15 April 2019; published online 5 June 2019

\begin{abstract}
Kurunthogai is a classical Tamil poetic masterpiece and it is the second book of Ettuthokai which is one of the Sangam literary works. The poems of Kurunthogai expresses the love life between men and women who lived during the Sangam age. Kurunthogai is a massive work written by many authors. The poems are written based on the five different landscapes namely, Kurinchi, Mullai, Marutham, Neythal, and Pālai. So, the poems contain much valuable historical information related to these landscapes. This paper proposes a templatebased Information Extraction (IE) framework for Kurunthogai which automatically extracts the names of flora, fauna, foods, vessels, and water bodies described in it. Furthermore, it extracts Noun Unigrams, Verb Unigrams, Adjective-Noun Bigrams, and Adverb-Verb Bigrams. Tamil Morphological Analyzer tool has been used to extract the N-grams. The state-of-art IE techniques have attempted to extract information from expository texts, whereas, the proposed IE framework extracts information from a literature-based text. The existing techniques extract information from monolingual texts, whereas, the proposed IE framework extracts information from bilingual texts. The proposed IE framework has achieved a precision of $88.8 \%$. The proposed framework can be applied for any literature type of texts and be used in various applications of Natural Language Processing.
\end{abstract}

Keywords. Kurunthogai; information extraction for Kurunthogai; template based IE; N-gram based IE; computational work for Tamil.

\section{Introduction}

Information Extraction (IE) is one of the most important applications of Natural Language Processing (NLP). With increasing web pages on the World Wide Web (WWW), the need for automatically extracting the important information from the unstructured pool of data has become quintessential. The IE systems also stand as one of the most important pre-processing factors for many NLP applications, such as Information Retrieval (IR) systems, Summary Generation systems, Intelligent Agents, etc. [1]. This paper proposes a unique IE framework which extracts the historical information from a Classical Tamil Literature called, Kurunthogai, which is the first of its kind [2].

The proposed methodology makes use of the TemplateBased Information Extraction technique to extract historical information from Kurunthogai. Template-based mining is used to extract data directly from a text if either the data and/or text surrounding the data form recognizable patterns. When the text pattern matches a template, the framework extracts information according to the rules specified in the templates. Though different techniques have been used for IE and knowledge discovery, template-based mining is the oldest technique and has proven to be efficient [3]. Since the Kurunthogai deals with nature, the lifestyle of the ancient Tamils, the templates used by the proposed framework is designed by focusing on these aspects [2]. The proposed approach makes use of the Universal Networking Language Knowledge Base (UNL-KB) to capture the semantically related terms [4]. Universal Networking Language (UNL) is a language-independent semantic representation that depicts a sentence as a semantic graph by converting each term into a Universal Word (UW) and each UW is semantically coupled using a UNL relation with other UWs [5]. UNL-KB is used to add semantics to each term. UNL-KB consists of hierarchically arranged 65436 UWs. The proposed approach makes use of this UNL-KB to extract the semantically related information from Kurunthogai when the term-based extraction fails to find an exact match between the features and the Kurunthogai words. Apart from historical information, the proposed approach extracts beautiful words that are present in Kurunthogai poems and are not currently used by the Tamils. This is done by extracting the N-grams using the Tamil Morphological Analyzer [6]. This tool is developed by the computational linguistic team of College of Engineering Guindy, Anna University. This tool can analyze the Tamil words and can identify 35 Parts of Speech categories such as Noun, Verb, Adjective, Finite Verb, and Infinite Verb. It completely works on the linguistic rules fed into it. 
Also, it identifies the free and the bound morphemes present in the given input word. The proposed approach makes use of this tool to identify the Parts-of-Speech categories namely, Nouns, Verbs, Adverbs, and Adjectives from which the $\mathrm{N}$-grams are identified. The $\mathrm{N}$-grams are nothing but the sequence of words or characters [7]. The proposed approach extracts word N-grams.

The proposed approach takes an initial attempt to make the ancient Tamil Literatures be available to the current IR systems. By doing so, these historical treasures can be taken to the current generation, which in turn will preserve their values for ages. The proposed approach also extracts information from English Translations of Kurunthogai. The main contributions of this paper are two-fold.

- Building an IE framework for a literature type of text.

- Building a bilingual- IE framework.

The rest of the paper is organized as follows. Section 2 describes the background of Kurunthogai. Section 3 describes the literature survey which focusses on various existing IE methods. Section 4 describes the proposed work. Section 5 describes the evaluation and performance analysis and section 6 gives the conclusion and future work.

\section{Background}

This section describes more about Kurunthogai and UNL.

\subsection{Kurunthogai}

Kurunthogai, a classical Tamil poem, is the second book of Ettuthokai, which is a Sangam literature written by many authors [2]. Nachinarkiniyar, a sixth century Tamil poet has annotated this work. Poems in Kurunthogai describe the lifestyle of the people lived in the Sangam age by portraying their love life, friendship and their culture. The poem given in Example 1 is one of the most famous poems in Kurunthogai written by an unknown poet. One way of preserving these historical works would be to make them computationally accessible. This paper makes such an attempt by proposing an IE framework through which, different information such as, food ate and the vessels used by the Sangam age people, flora and fauna lived during that period and also about the nature bodies that are described in Kurunthogai poems are extracted automatically. Kurunthogai has many beautiful Tamil words which can be useful for building automatic lyric generation systems which are gaining importance in recent days [8]. Tamil literature has a 2,200-year-old history and the Tamil language has transformed a lot in terms of script and the way it is spoken [9]. The current Tamil speaking generation may not be aware of the words used in the Sangam literature. Certain film songs and poetries do contain the words used in Sangam literature which increases the poetic value and adds beauty to it. The proposed system aims at extracting the information from Kurunthogai and this information can also be a source of both historical knowledge and also as a literature word knowledge that can be used in lyrics or poetry by the current generation. Example 1 shows a song from Kurunthogai in Tamil and its translation in English.

Example 1: An example song from Kurunthogai and it's English Translation.

$$
\begin{aligned}
& \text { நெய்தல் புள்ளும் புலம்பின; பூவும் } \\
& \text { கூம்பின;கானலும் புலம்பு நனி உடைத்தே; } \\
& \text { வானமும்,நம்மே போலும் மம்மர்த்து } \\
& \text { ஆகி,எல்லை கழியப் புல்லென்றன்றே;இன்னும் } \\
& \text { உளெனே தோழி! இந்நிலை தண்ணிய கமழும் }
\end{aligned}
$$$$
\text { ஞாழல் }
$$$$
\text { தண்ணம் துறைவற்கு உரைக்குநர்ப் பெறினே }
$$$$
\text { வரைவிடை முனிந்து கிழத்தி தோழிக்கு }
$$$$
\text { உரைத்தது . }
$$$$
\text { பெருங்கண்ணன் பாடல் }
$$

\section{English Translation:}

Dark is everywhere. As my mind is all dark the birds cry over this darkness. The flowers are not blooming. The light in the littoral land is waning. The sky steals the darkness. All are imitating my mind. Still, I am living. Is there anybody who can convey my situation to my man who is as warm as the NJA: ZAL flower. The lady is seeking her friend's help.

\section{$2.2 U N L$}

UNL is a semantic representation that captures the semantic relation between the words. The words are represented as concepts. The relations are called as UNL relations and the concepts as UWs. There are $46 \mathrm{UNL}$ relations defined by the UNDL foundation [5]. The Enconverter is a system that converts the natural language text to a UNL expression and a Deconverter is a system that converts a UNL expression to a natural language text. The UNL expression can be visualized as a graph like structure where each node is represented by the UWs and each edge between the nodes are formed by the UNL relations. A UW consists of two parts namely, the headword and the semantic constraint. The headword is merely the root of the natural language word and the semantic constraint expresses the context in which the word is used. For example, the word, "apple" 


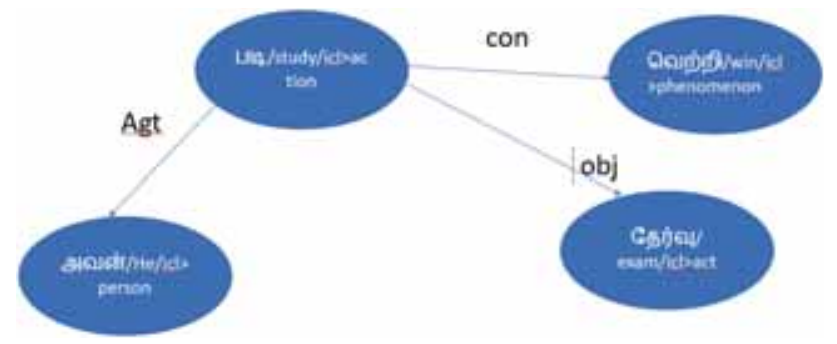

Figure 1. The UNL graph for Example 1.

would be represented as, "Apple(iof $>$ fruit)" in a sentence in which it gives the meaning of the fruit, whereas, the Mac apple laptop would be represented as, "Apple(icl>computer)". These semantic constraints are taken from the UNL-KB after analyzing the contexts of the words in a sentence. The rules are formed to attach the correct UNL relation between the UWs. The UNL representation is a language-independent representation and remains the same for all languages. The respective bilingual UW dictionary needs to be supported for framing the UWs and the language dependent rules are to be framed to relate the UWs using UNL relations. Example 2 gives an example of Tamil sentence for which the UNL graph is given in figure 1.

\section{Example 2:}

அவன் படித்ததனால் தேர்வில் வெற்றி
பெற்றான்

English Translation: He passed in the exam because he studied well.

The UNL relation, Agt represents the agent; con represents the condition and the obj represents the object.

The proposed approach makes use of the UNL-KB to match and extract the semantic information from Kurunthogai which is explained in section 3 .

\section{Literature survey}

Since the proposed framework focusses on IE systems and computational analysis of Kurunthogai, the literature survey has been done on the existing works on IE and computational works done on Tamil literature.

The IE system proposed by Mooney et al extracts information from biomedical abstracts, job announcements and product descriptions [10]. They have used data mining algorithms such as SOFTRISE and SOFT APRIORI to extract the information from the unstructured data.

Ahmed et al have used an N-gram based approach to extract the information from the web documents [11]. They have used the Latent Semantic Indexing (LSI) to extract the $\mathrm{N}$-gram information. LSI is a widely used indexing mechanism used by the search engine to identify the closely related indices in large document-term matrix space. $\mathrm{N}$-gram based LSI has proved to give better results in IR systems.

Nathanael Chambers et al have come up with a templatebased IE method which identifies the templates automatically from the text patterns without knowing the structure in advance [12]. This method has been used to extract linked events.

Alan Ritter et al have proposed an IE system which extracts the calendar of events from tweets [13]. The information extracted is independent of the domain and the events are also classified based on the keyword phrases that appear on them.

It can be observed that the current IE techniques aim at extracting the information from the large collection of unstructured web data. The proposed system differs from the state-of-the-art systems by extracting information from a literature type of text. The greatest challenge involved in handling a classical literature type of texts for languages like Tamil is the availability of lexical resources. The transformation that has happened in the Tamil language which makes it difficult to process the words that are not currently used. The proposed approach makes use of bilingual word mappings which is collected from various online resources to handle both English and Tamil versions of Kurunthogai.

From the Tamil literature point of view, many computational works have been done in recent times. Elanchezhiyan et al have designed a concept-based search engine for Thirukural, which is one of the popular literature of Tamil language written by Thiruvalluvar [14]. Subalalitha and Ranjani have proposed a unique indexing technique by using the concepts of Nanool, which is a grammar masterpiece written by Pavananthi Munivar [15]. Subalalitha and Poovammal have come up with a framework to automatically construct a bilingual dictionary for Thirukkural by using the Tamil and English translations of Thirukkural [16]. Inspired by these works, this paper attempts an IE framework for Kurunthogai which can aid in unveiling the historical information present in the Sangam literature.

The next section describes the proposed IE framework.

\section{Proposed work}

\subsection{Architecture and working}

Figure 2 shows the architecture of the proposed IE framework. The Kurunthogai songs in both Tamil and English are given as inputs to the proposed IE framework. The two major types of information extracted are template-based information and $\mathrm{N}$-gram based information.

The next section describes the template-based information extraction. 


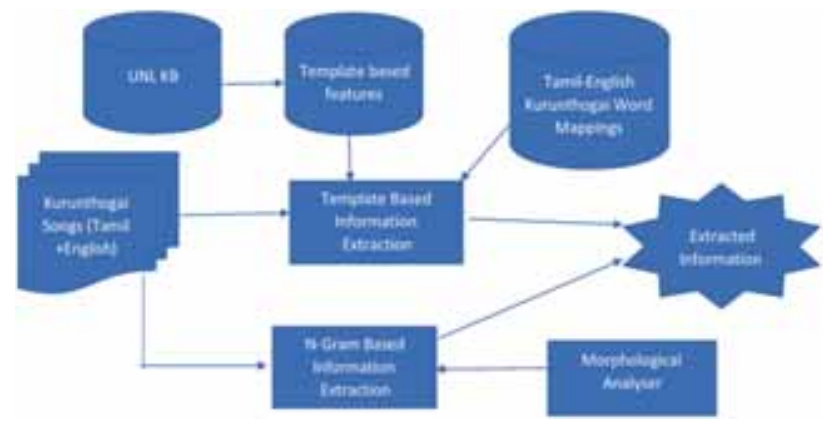

Figure 2. Architecture of the proposed IE framework.

4.1a Template-based information extraction: The framework uses templates to extract information. Since the features used by the templates are in English, Tamil to English bilingual word mappings is used to extract the information from the Tamil Kurunthogai songs by translating each word using bilingual mappings. The features can also be in Tamil. Since the proposed approach extracts information from both English and Tamil Kurunthogai songs and the UNL-KB features are in English, the features are represented in English. About 10,000 Tamil-English word mappings for literature specific words have been used which are collected from various online resources. The templates have been designed to extract the information from Kurunthogai using two types of features, namely term-based features and UNL$\mathrm{KB}$ features which are listed in table 1. The term-based features look for an exact match between the input words and the features. For instance, in the word, “கமழ்பூ fragrant flower", the feature, "flowers" is present and will be extracted by the term-based features, whereas, in the word, "பசுங்கலம் போல - like an unfired clay pot", the feature, "vessel" is not present. In such cases, the UNL$\mathrm{KB}$ is used, which converts the word into a UNL concept by adding a semantic constraint to it. The semantic constraints are added to each bigram present in the word mappings. In this case, the semantic constraint would be, "icl>vessel" which will be matched by the UNL-KB features. The UNL-

Table 1. Template-based features.

\begin{tabular}{|c|c|c|}
\hline \multirow[b]{2}{*}{ Templates } & \multicolumn{2}{|c|}{ Features } \\
\hline & Term based features & UNL KB features \\
\hline Flora Info & $\begin{array}{l}\text { "plant/plants", } \\
\text { "flower/flowers" }\end{array}$ & "icl $>$ plant", iof $>$ plant \\
\hline $\begin{array}{l}\text { Fauna } \\
\text { Info }\end{array}$ & $\begin{array}{l}\text { "animal", "bird", } \\
\text { "fish" }\end{array}$ & $\begin{array}{c}\text { "icl }>\text { animal", iof }>\text { animal, } \\
\text { icl }>\text { bird, icl }>\text { fish }\end{array}$ \\
\hline Food Info & "food" & "iof $>$ food", icl $>$ food \\
\hline $\begin{array}{c}\text { Vessels } \\
\text { used }\end{array}$ & "vessel" & $\begin{array}{c}\text { Obj>thing, icl>vessel, } \\
\text { obj>container }\end{array}$ \\
\hline $\begin{array}{l}\text { Water } \\
\text { Bodies }\end{array}$ & "water", "river" & "icl > river" \\
\hline
\end{tabular}

KB features aids in extracting the words that do not explicitly match with the term-based features.

Example 3 shows the template-based information extracted by the proposed framework.

\section{Example 3:}

கமழ்பூ - fragrant flower

பூவென - like signature flowers for madal ēruthal

எருக்கங் கண்ணியும் - erukkam flower garland

உள்ளூர்க் குரீ - sparrow in town

பசுங்கலம் போல - like an unfired clay pot

இருங்கழி - dark/huge backwaters

The proposed work also extracts four types of N-grams, namely, Verb Unigrams, Noun Unigrams, Adjective-Noun Bigrams and Adverb-Verb Bigrams which are explained in the next section.

4.1b N-Gram extraction: The N-grams are extracted only from the Tamil Kurunthogai songs. The Parts-of-Speech of the words present in the Kurunthogai songs in Tamil are identified by the Tamil morphological analyzer [6]. The analyzer identifies, Nouns, Verbs, Adjectives, and Adverbs. The Nouns and Verbs form the Unigrams, whereas, the Adjective-Noun and the Adverb-Verb pairs form the Bigrams. Example 4 shows the Adjective -Noun Bigrams extracted by the proposed framework.

\section{Example 4:}

$$
\begin{aligned}
& <ச ை வ>\text { : } \\
& \text { சைவ }<\text { Adjectival Noun \& } 106>\text { count }=0 \\
& <<\text { wc }> \\
& <\text { சித்தாந்த>: } \\
& \text { சித்தாந்த < Adjectival Noun \& } 106>\text { count=0 } \\
& <<\text { wc }>
\end{aligned}
$$

The main aim of this $\mathrm{N}$-gram extraction is to bring out the beautiful words that were used in the Kurunthogai songs during the Sangam age and are not used by the current generation.

The template-based extraction extracts information from both Tamil Kurunthogai songs and English songs. The Tamil-English mappings aid to extract the Tamil information and these mappings are generated from various TamilEnglish parallel Sangam Literature songs. The information from the English Kurunthogai song can directly be handled by the templates. The $\mathrm{N}$-gram information aims only at extracting the Tamil word pairs. 
Table 2. Performance analysis of the proposed IE framework.

\begin{tabular}{lcccc}
\hline Sl.no. & $\begin{array}{c}\text { Type of information } \\
\text { extracted }\end{array}$ & $\begin{array}{c}\text { Total no. of information } \\
\text { extracted }\end{array}$ & $\begin{array}{c}\text { Total no. of correctly extracted } \\
\text { information }\end{array}$ & $\begin{array}{c}\text { Precision achieved } \\
(\%)\end{array}$ \\
\hline 1 & Flora & 82 & 60 & 73 \\
2 & Fauna & 32 & 25 & 78 \\
3 & Vessels & 11 & 11 & 100 \\
4 & Water Bodies & 60 & 55 & 91.6 \\
5 & Food Information & 44 & 2365 & 93 \\
6 & Noun Unigrams & 2365 & 1234 & 92 \\
7 & Verb Unigrams & 1234 & 26 & 90 \\
8 & Adjective-Noun Bigrams & 26 & 5 & 91 \\
9 & Adverb-Verb Bigrams & 5 & 88.8 & \\
Average Precision & & & \\
\hline
\end{tabular}

\section{Evaluation and performance analysis}

The evaluation has been done for about 200 Kurunthogai songs out of 400 songs which are both in Tamil and English. The proposed model can be tested for the entire 400 songs when the size of the bilingual Tamil-English word mappings is increased. The human judgement has been used to evaluate the proposed approach and "precision" has been used as the evaluation parameter which is defined as shown in Eq. (1).

$$
\begin{aligned}
\text { Precision }= & \text { Number of correct Information extracted } / \\
& \text { Total number of Information Extracted }
\end{aligned}
$$

The precision values for each type of information extracted is listed in table 2.

The precision measurement is done by six experts who have knowledge in Kurunthogai. $88.8 \%$ precision is the average percentage. It can be observed that the precision for all the N-grams extracted is higher than that of templatebased information. This is due to the fact that the precision of $\mathrm{N}$-grams relies on the precision of the Tamil Morphological Analyser which identifies the Parts of Speech (POS) information precisely. The precision of the information extracted through the templates mostly relies on the match quality between the template features and the information extracted. The more is the length of the information, the more is the chance of it falling into the wrong category. For instance, the information, "பூ இடைப்படினும்" which means even if a flower comes in between" is categorized under flora as it contains the word flower. Understanding the deeper semantics from the information extracted by identifying the Named Entities (NE) can improve the precision [17]. The identification of NEs and classifying them by using Machine Learning Algorithms like Support Vector Machines (SVM) can aid in extracting a variety of information, such as medicines, weapons, etc. Also, the TamilEnglish word mappings can automatically be generated from the Tamil-English parallel Kurunthogai songs [16]. The proposed approach is a primitive model to initiate the process of analyzing ancient literature.

\section{Conclusion and future work}

An IE framework for Kurunthogai has been proposed using the template-based and $\mathrm{N}$-gram based features. Kurunthogai is a Tamil literature masterpiece belonging to the Sangam age [2]. This paper has attempted to extract historical information in Kurunthogai. Information about the natural resources, such as flora, fauna, water bodies, foods eaten and the vessels used during the Sangam age are extracted. This historical information unveils the culture of Sangam age. The N-grams such as Nouns, Verbs, Adjective-Noun pairs, and Adverb-Verb pairs are extracted. These N-grams help to unveil the beautiful Tamil words used in Kurunthogai to the current generation. These words can be used by the current lyricists to use these ancient words in the songs penned by them. Also, the proposed approach can help in building automatic lyric generation systems [8].

The proposed approach can be applied to extract information from any Tamil kinds of literature that are like Kurunthogai such as, Natrinai which also describes the life of people lived during Sangam age [2]. Also, the proposed approach can be extended to extract information from any language text provided with the suitable features, English translations, and bilingual word mappings. The proposed rule-based approach is an initiative which needs to be enhanced with more templates. Automatic learning of templates and augmenting the initial set of features using neural networks and machine learning algorithms can make the proposed approach completely language-independent and lexical resource-independent [18]. This can aid in unveiling the ancient information hidden in the literature of many languages to the current generation. 


\section{References}

[1] Ropero J, Gomez A, Carrasco A and León C 2012 A fuzzy logic intelligent agent for information extraction: introducing a new fuzzy logic-based term weighting scheme. Expert Syst. Appl. 39(4): 4567-4581

[2] Mudaliyar A S and Cintamani A 1983 An encyclopedia of Tamil Literature, Reprinted by Asian Educational Services: New Delhi

[3] Gaizauskas R and Wilks Y 1998 Information extraction: beyond document retrieval. J. Doc. 54(1):70-105

[4] Verma N and Bhattacharyya P 2004 Automatic lexicon generation through WordNet. GWC, 226

[5] Uchida H and Zhu M 2001 The universal networking language beyond machine translation. In: International Symposium on Language in Cyberspace, Seoul, pp. 26-27

[6] Anandan P, Saravanan K, Parthasarathy R and Geetha T V 2002 Morphological analyzer for Tamil. Proceedings of ICON

[7] Brown P F, Desouza P V, Mercer R L, Pietra V J D and Lai J C 1992 Class-based n-gram models of Natural Language. Comput. Linguist. 18(4): 467-479

[8] Sridhar R, Ganga K and Prabha G D 2014 Automatic Tamil lyric generation based on ontological interpretation for semantics. Sadhana 39(1): 97-121

[9] Abraham S A 2003 Chera, Chola, Pandya: using archaeological evidence to identify the Tamil Kingdoms of early historic South India. Asian Perspect. 207-223

[10] Mooney R J and Bunescu R 2005 Mining knowledge from text using information extraction. ACM SIGKDD Explor. Newslett. 7(1): 3-10
[11] Güven A, Bozkurt Ö Ö and Kalıpsız O 2006 Advanced information extraction with n-gram based LS. In: Proceedings of World Academy of Science, Engineering and Technology, pp. 13-18

[12] Chambers N and Jurafsky D 2011 Template-based information extraction without the template. In: Proceedings of the 49th Annual Meeting of the Association for Computational Linguistics, pp. 976-986, Portland, Oregon

[13] Ritter A, Mausam, Etzioni O and Clark S 2012 Open domain event extraction from twitter. In: Proceedings of the 18th ACM SIGKDD international conference on knowledge discovery and data mining, Beijing, China

[14] Elanchezhiyan K, Geetha T V, Parthasarathi R and Karky M 2011 Kuralagam-concept relation based search engine. In: Tamil Internet Conference, pp. 19-23

[15] Subalalitha C N and Ranjani P 2013 A unique indexing technique for discourse structures. J. Intell. Syst. 23(3): 231-243

[16] Subalalitha C N and Poovammal E 2018 Automatic bilingual dictionary construction for Tirukural. Appl. Artif. Intell. 32(6): 558-567

[17] Manipoonchelvi P and Muneeswaran K 2014 Multi region-based image retrieval system. Sadhana 39(2): 333-344

[18] Lin C, Miller T, Dligach D, Amiri H, Bethard S and Savova G 2018 Self-training improves recurrent neural networks performance for temporal relation extraction. In: Proceedings of the Ninth International Workshop on Health Text Mining and Information Analysis, pp. 165-176 